\title{
Food fortification: good to have or need to have?
}

\author{
A. Verster ${ }^{1}$
}

SUMMARY Only very small quantities of vitamins and minerals are needed for human health but deficiencies can have disproportionately large, often life-threatening, effects. Micronutrient deficiencies lead to a vicious cycle of malnutrition and infection in poorly nourished populations. Fortification of staple foods is the cheapest, most efficient and most effective way to supply large populations with essential micronutrients. This paper reviews the case for fortification of flour supplies with iron and folic acid and concludes that it is the best way to provide daily doses of these nutrients to populations in developing countries, especially for women of child-bearing age.

\section{Introduction}

Vitamins and minerals, often called micronutrients, are essential for human physical and mental health and survival. Only very small quantities are needed, but deficiencies can have disproportionately large, often life-threatening, effects.

Only a teaspoon of iodine is needed for a lifetime, but iodine deficiency causes irreversible mental retardation. Only $400 \mu \mathrm{g}$ a day of folic acid protects an unborn child from neural tube defects such as spina bifida or from anencephaly. Just $6-12 \mathrm{mg}$ of iron a day is enough to help a child develop to its full mental and physical potential. And with $450 \mu \mathrm{g}$ retinol per day, a child will have a strong immune system and will not be at-risk of losing its eyesight.

Vitamins and minerals are found in many foods, and in principle, a "balanced" diet, based on a combination of plant and animal foods, as advised by various dietary guidelines, should provide sufficient vitamins and minerals, except for iodine.

Yet deficiencies of many important vitamins, such as vitamin $\mathrm{A}$ and vitamin $\mathrm{D}$, and minerals such as iron, calcium, zinc and iodine are widespread in many parts of the world. Iodine is a special case, in that this deficiency has geophysical origins, affecting all countries where iodine has been leached out of the soil through flooding and glaciers or where there are tertiary sedimentations that never contained iodine. The high prevalence of anaemia in many parts of the world (Figure 1) suggests that iron deficiency is common and persistent. Vitamin A deficiency is associated with 800000 deaths worldwide each year. Data from the United Kingdom shows that Vitamin A deficiency is a problem not only in developing countries but also in the industrialized world, albeit less severe. Therefore, a so-called balanced diet may not be so widely consumed as expected, or the yield in vitamins and minerals from these diets may not be as high as previously thought.

This paper will review the case for food fortification with iron and folic acid, and suggest that flour fortification is not just good to have: it is essential for the health of populations.

${ }^{1}$ WHO Senior Technical Adviser, Global Alliance for Improved Nutrition, World Health Organization, Geneva, Switzerland.

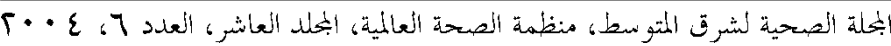




\section{Making the case for micronutrients}

More than 2 billion people in the world today, nearly all of them in developing countries, suffer from nutritional deficiencies, mainly the lack of micronutrients such as iron, vitamin A, iodine and folic acid [1]. Overtly, micronutrient deficiencies can cause irreversible damage to health such as blindness, or contribute to maternal death during childbirth, or to infant and child death as a result of otherwise common childhood illnesses. In most cases, however, micronutrient deficiency causes weakness and greater susceptibility to illness. This vicious cycle of malnutrition and infection, ultimately leading to death, is well known and feared in poorly nourished populations.

Yet the wider impact of micronutrient deficiency on a nation's economy is unknown or unacknowledged among government leaders, politicians and economists, despite the fact that it traps not only individuals and communities but also entire countries in a cycle of poor health, poor educability, poor productivity and persistent poverty. Added to this is the individual human suffering of families who lose a mother in childbirth due to iron deficiency or who bear the burden of caring for a child born retarded because of maternal iodine deficiency during pregnancy, or of whole communities that suffer weakness and ill health that prevents their crossing the threshold out of poverty.

It follows that ending micronutrient deficiency can provide the foundation for the elimination of poverty and for sustainable economic progress among poor countries by preventing illness and death and by helping populations to become healthier, more intelligent, more educated and more productive. Sufficient intake of vitamin A, essential to the human immune system, would prevent almost a quarter of child deaths in areas of high deficiency, as well as preventing nutritional blindness among children and adults. Universal salt iodization would contribute considerably to the brain development of children in areas of deficiency, adding up to 13 IQ (intelligence quotient) points to their mental capacity

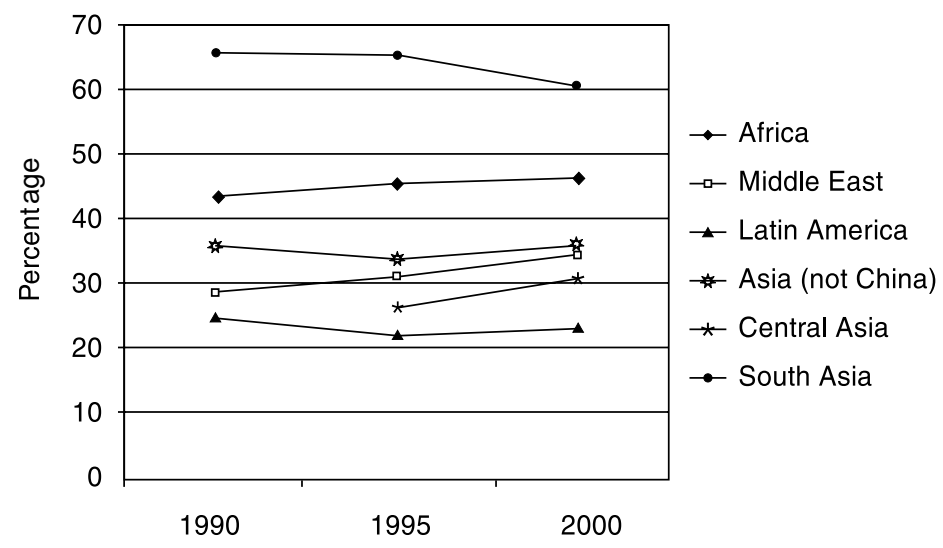

Figure 1 Estimated prevalence of anaemia in non-pregnant women in 6 world regions over time. Source [8]

البحلة الصحية لشرق المتو سط، منظمة الصحة العالمية، البخلد الصاشر، العدد لج، ع • +. 
and eliminating the world's greatest cause of preventable mental retardation. Ending iron deficiency would reduce absenteeism due to illness and thus improve adults' productivity. It would also improve children's concentration and memory and therefore their ability to learn.

In 1994, the World Bank estimated that interventions to end micronutrient malnutrition were among the most cost-effective investments in the health sector. The global cost of ending micronutrient deficiency was estimated by the Bank at about US\$1 billion a year [2]. Failure to act in a country where micronutrient malnutrition exists can waste as much as $2 \%-3 \%$ of the gross domestic product (GDP) [3]. Acting to solve the problem comprehensively and sustainably would cost less than $0.3 \%$ of GDP. Clearly there are economic as well as ethical imperatives for effective action to provide large populations with micronutrients.

The first of the United Nations Millennium Development Goals, eradicating extreme poverty and hunger, is unlikely to be achieved in the absence of well-nourished, intelligent, healthy, productive populations. In fact, 5 of the Millennium Development Goals can be positively influenced by food fortification, as shown later in this paper. Fortification of staple food supplies, linked with other initiatives such as agribusiness or small business development or poverty reduction strategies, has tremendous potential to reduce poverty.

There is a cost to beginning this work, but the economic and social payoffs from micronutrient programmes, according to the World Bank, reach as high as 84 times the programme costs. Recently a group of leading economists (the Copenhagen Consensus) proclaimed that the addition of micronutrients into food was the second most effective intervention in favour of development [4].

\section{Food fortification}

The success of programmes of universal salt iodization in eliminating iodine deficiency disorders clearly demonstrates the effectiveness of food fortification as a vehicle for improving micronutrient status. Fortifying staple foods can be done in many countries on a national scale. It is the cheapest, most efficient and most effective way to supply large populations with essential micronutrients. Bringing the technology to fortify staple foods such as flour, salt and oil and condiments such as soy sauce to developing countries is a costeffective solution to micronutrient deficiency, especially when undertaken in tandem with broader national strategies that improve overall nutritional status.

The cost of fortifying staple foods is not high, and food fortification does not require people to change their existing dietary habits. Because staple foods such as bread or noodles are used daily, and are part of the diets of even the poorest, fortification has the potential in a short period of time to reach millions of people around the world with micronutrients on a daily basis.

\section{Iron fortification of flour}

Iron deficiency is not easily detected, although its consequences are important. Most often, anaemia is taken as a proxy indicator for iron deficiency, especially in parts of the world where anaemia is largely caused by iron deficiency. In the Eastern Mediterranean Region (EMR), for instance, measurements in several countries suggest that only $6 \%-10 \%$ of all anaemia is due to

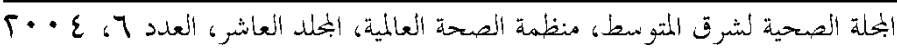


causes other than iron deficiency. The prevalence of anaemia is generally high among women of childbearing age and young children in all countries of the Region. Anaemia, however, is not synonymous with iron deficiency. In fact, iron deficiency is generally present in individuals long before haemoglobin values fall. First the body's iron stores are diminished and only then is haemoglobin production affected.

In the past, pregnant women were the main targets for anaemia control programmes. It was long believed that the main effects of iron deficiency, especially when anaemia is already present, were a risk of maternal death in childbirth and lowbirth-weight infants. This, coupled with the especially high iron requirements of pregnancy, means that pregnant women were considered most at risk. There is increasing evidence, however, that iron deficiency has less obvious, developmental effects on children; they may be less intelligent, scoring up to 10 points lower on standard IQ tests, their school performance may be below normal and their future productivity may be diminished. Now that our understanding of the effects of iron deficiency has grown, programmes have become increasingly targeted towards prevention of iron deficiency, rather than treatment of anaemia. Combined approaches of supplementation with iron tablets, public health measures such as de-worming and nutrition education to improve the iron content of the diet have become popular.

Brain development takes place in the first trimester of pregnancy: a time when a woman may not yet know that she is pregnant. She will not yet have visited a health facility for prenatal care, and thus will not have received iron supplements. Yet, this is the time when iron is necessary for the development of the unborn child's brain. It is therefore imperative to ensure that a woman's iron status is optimal before she gets pregnant. The only feasible way is to ensure that her daily diet contains sufficient iron.

Unfortunately, iron is a fickle nutrient. There are 2 different types of iron in food: haem iron, found in red meat, seafood and poultry, and non-haem iron found in grains, legumes, nuts and other plant foods. About $40 \%$ of the iron in animal food is in the form of haem iron, while the rest and almost all the iron in plant foods is in the nonhaem form that is less well absorbed by the body. The bioavailability of non-haem iron is influenced by factors in the diet that either enhance or inhibit absorption, as shown in Figure 2.

The bioavailability of iron in the diet varies therefore according to the type of diet, but rarely exceeds more than $5 \%-10 \%$ of the total iron consumed. It is very difficult therefore to get enough iron from the normal diet, even in developed countries. In the EMR, consumption of wheat flour is very high; daily intakes of 300-400 $\mathrm{g}$ of bread per day are reported from several countries. The wheat grain, in its un-milled form or if milled into whole-wheat flour, contains good quantities of iron. This iron, however, has a low bioavailability as it is bound to the phytates that are intrinsic to wheat. On the other hand, white flour contains much less phytate, but unfortunately does not contain high levels of iron either.

Fortifying wheat flour with iron, preferably in the form of ferrous sulfate or ferrous fumarate, which are both well absorbed, makes it possible to provide a large amount of additional iron to the diet of women, and indeed the entire population. If a woman consumes a reasonable amount of bread every day, the additional iron from fortification changes her iron status from deficient to sufficient, thus protecting the

الجملة الصحية لشرق المتو سط، منظمة الصحة العالمية، البملد العاشر، العدد ج، ع +. 


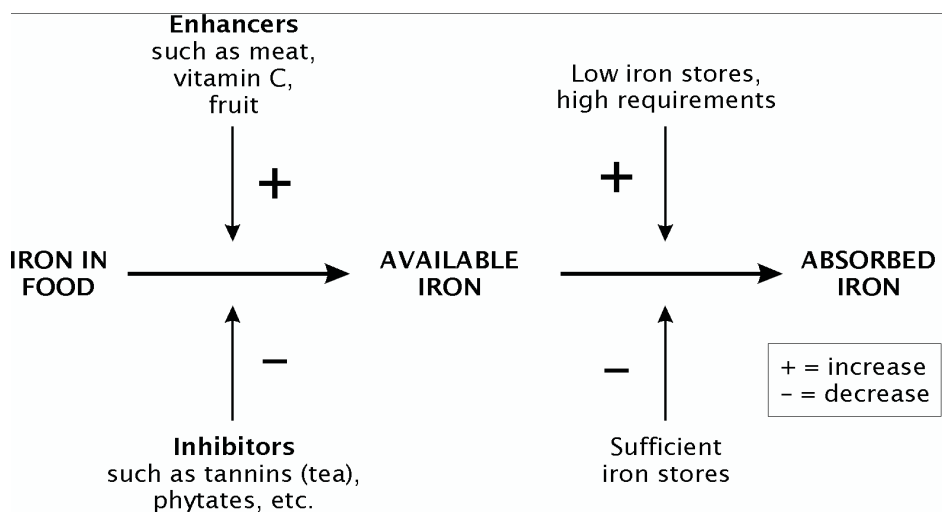

Figure 2 Dietary factors that affect the bioavailability of non-haem iron by enhancing $(+)$ or inhibiting (-) absorption. Source: A. Verster

brain of any child she conceives. The United Nations Food and Agriculture Organization estimates that flour fortification can contribute from $50 \%$ to over $75 \%$ of the daily iron requirement in countries of EMR (Figure 3).

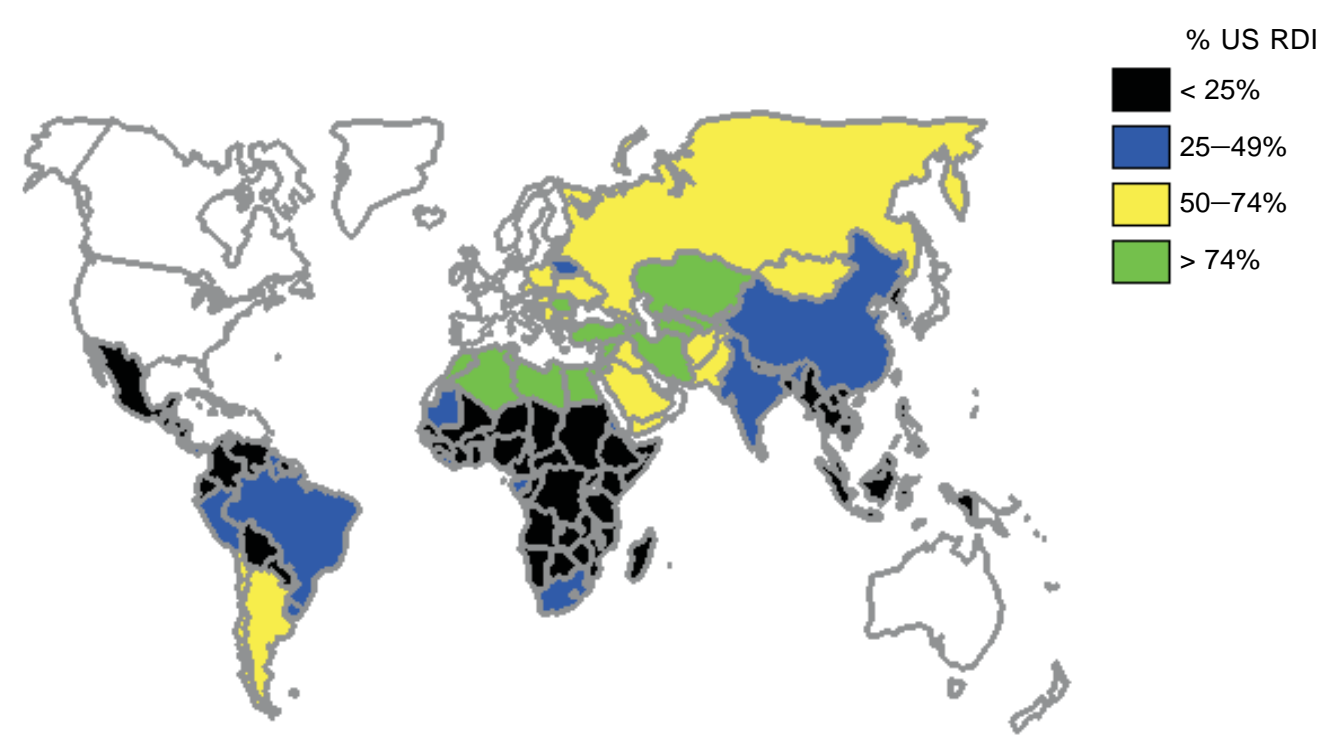

Figure 3 Potential benefit of flour fortification to iron intake. Contribution to \% iron recommended daily intake if 30 parts per million of iron were added to wheat flour. Source: J.Bagriansky and K. Sullivan (unpublished data: http://www.sph.emory.edu/wheatflour/ Training/Data_Evaluation/Other/Benefitcalc.xls)

البحلة الصحية لشرق المتو سط، منظمة الصحة العالمية، البكلد العاشر، العدد ج، ع • 


\section{Folic acid fortification of flour}

Flour fortification is already in place in several counties of EMR (Saudi Arabia, Oman, Bahrain, Qatar, United Arab Emirates, Jordan and Morocco). In the early days of flour fortification, WHO-EMR included folic acid in its fortification premix recommendations, mainly because of the role of folic acid in anaemia. Over time, more evidence has become available of the preventive effect of folic acid on birth defects, especially neural tube defects such as spina bifida and anencephaly. Clearly, WHOEMR was ahead of its time with the recommendation to add at least iron and folic acid to wheat flour. Recently published scientific research shows that folic acid fortification of wheat flour has led to a decline in the incidence of neural tube defects in several countries that have studied these effects, notably Chile, Costa Rica [5] and Canada [6] (Figure 4). The Canadian study, which was carried out in women aged 1944 years and on seniors in Newfoundland, did not find any masking of vitamin $B_{12}$ deficiency by folic acid fortification. The researchers concluded that mandatory fortification of wheat flour with folic acid should continue in Canada at the current levels.

\section{Food fortification and the Millennium Development Goals}

According to the Global Alliance for Improved Nutrition, food fortification contributes to the achievement of 5 of the United Nations Millennium Development Goals [7].

By ensuring that a population's food includes essential vitamins and minerals, individual productivity is improved, which will contribute to poverty alleviation, thus contributing to Goal 1 - eradicate extreme
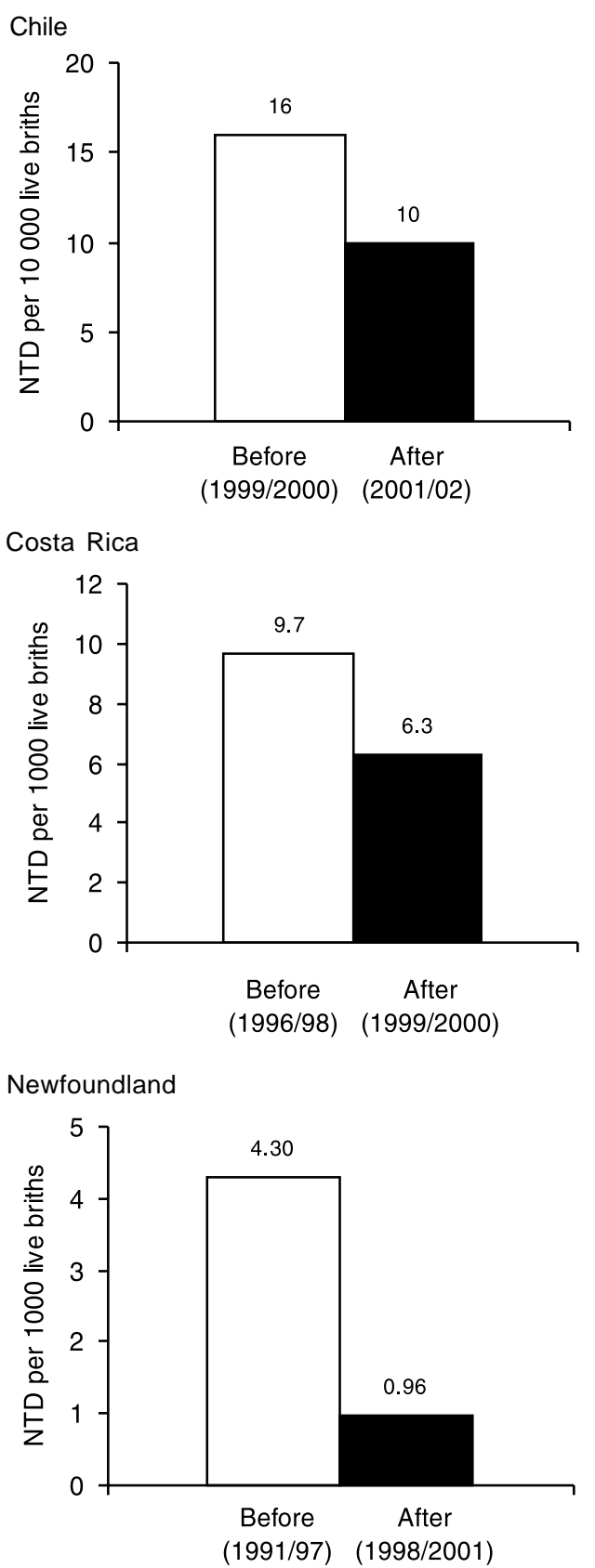

Figure 4 Annual rates of neural tube defects (NTD) in Chile [5], Costa Rica [5] and Newfoundland [6] before and after fortification of flour with folic acid 
poverty and hunger. Fortification programmes that reach the very young can improve cognitive function and intellectual capacity, enabling children to do better in school, thus contributing to Goal 2 achieve universal primary education.

Better nutrition, including the reduction of iron deficiency and anaemia among adolescent girls, is a prerequisite for improving women's physical and mental capacity and their ability to participate as equal citizens. Fortification of staple foods that are commonly consumed by young women and girls is one way to contribute to Goal 3 promote gender equality and empower women.

Improving the nutritional status of infants and children through fortifying the staple foods used to prepare complementary foods, and fortifying appropriate complementary foods themselves, is essential to the achievement of Goal 4 - reduce child mortality.

Under-nutrition and anaemia of girls and women contribute significantly to maternal morbidity and mortality and to low birth weight. Significant improvements in the macro- and micronutrient status of girls, adolescents and women through fortification as well as other essential strategies such as micronutrient supplementation, dietary diversification and public health measures are needed to achieve Goal 5 improve maternal health.

\section{Conclusion}

Fortification with iron and folic acid is the best way to provide daily doses of these essential micronutrients to women before they get pregnant and in the early months of pregnancy when brain development and neural tube developments take place. It is the most effective approach in societies where women do not plan their pregnancies. Fortification of flour with iron and folic acid is therefore not only good to have: it is an absolute necessity.

\section{References}

1. World health report 2002. Reducing risks, promoting healthy life. Geneva, World Health Organization, 2002.

2. Enriching lives: overcoming vitamin and mineral malnutrition in developing countries. Washington DC, World Bank, 1994.

3. United Nations Children's Fund/The Micronutrient Initiative. Vitamin and mineral deficiency. A global progress report. New York, UNICEF, 2003.

4. Copenhagen consensus-vision and facts. Copenhagen, Copenhagen Consensus, 2004 (http://www. copenhagenconsensus.com, accessed 28 February 2005).
5. Flour fortification with iron, folic acid and vitamin B12. Washington DC, Pan American Health Organization, 2004.

6. Liu $\mathrm{S}$ et al. A comprehensive evaluation of food fortification with folic acid for the primary prevention of neural tube defects. BMC pregnancy and childbirth, 2004, 4:20.

7. Fortifying the fight against poverty. Strategic plan 2004-2007. Geneva, Global Alliance for Improved Nutrition, 2003.

8. Micronutrient Initiative special section reprint. Food and nutrition bulletin, 2005, 26(1).

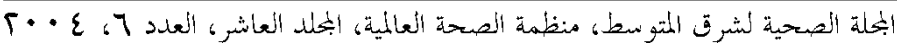

